\title{
Mouse model reveals how appendicitis protects against ulcerative colitis
}

Appendicitis and appendectomy (AA) at a young age seem to be associated with a reduced risk of developing ulcerative colitis. New findings from a mouse model suggest that autophagy-related genes could be involved in this association.

Seven so-called autophagy genes are known to be linked with IBD. "Therefore, we were interested in knowing whether the modulation of other autophagy genes could limit colitis pathology," explains lead author Rajkumar Cheluvappa.

The researchers developed a mouse model of AA that demonstrated protection against experimental colitis and then analysed gene expression in the distal colon of the mice. The expression of three autophagy genes was slightly upregulated, but no variation was seen in the expression of the 25 other autophagy genes examined.

However, enrichment analysis of groups of genes (gene sets) revealed that 74 gene sets that are associated with the 28 autophagy genes examined were downregulated and five gene sets were upregulated (associated with VPS15, $L A M P 2, L C 3 A, X B P 1$ and ULK1). The team conclude that AA induces profound suppression of autophagy in the distal colon. This suppression might result in reduced antigen processing, which would lead to reduced cross-reactive immunity between mice and self-antigens and thus a decreased risk of ulcerative colitis.

With a view to developing new therapies, Cheluvappa and colleagues would like to extend their findings using competitive antagonism in their model, as well as gene-knockout mice. "Autophagy, owing to its foundational role in gut homeostatic maintenance, is an attractive therapeutic target," they conclude.

\section{Claire Greenhill}

Original article Cheluvappa, R. et al. Autophagy suppression by appendicitis and appendectomy protects against colitis. Inflamm. Bowel Dis. doi:10.1097/MIB. 0000000000000034 